Reprod. Nutr. Dévelop., 1980, 20 (5 B), 1641-1644.

\title{
Contribution à la description de la prise alimentaire de la chèvre
}

\author{
par P. MORAND-FEHR, J. HERVIEU, D. SAUVANT \\ Laboratoire de Recherches de la Chaire de Zootechnie \\ Institut National Agronomique Paris-Grignon \\ 16, rue Claude-Bernard, 75231 Paris Cedex 05.
}

Summary. A contribution to the description of feed intoke in goats.

Four adult goats were given lucerne hay ad libitum and had access to water, salt stone and the litter straw. They were housed in a box and were completely free to move.

Their meals consisted of the main behavioural activity of hay intake and other activities such as salt-stone licking, water drinking and straw intake. Ingestion was relatively slow because the goats took a long time to choose what they ingested. They chose the hay with more leaves and less stems which had a higher nutritive value.

La chèvre a la renommée de choisir de façon plus sélective que les autres ruminants ce qu'elle ingère, que ce soit dans des conditions d'alimentation intensive (Morand-Fehr et al., 1977) ou sur parcours (Davendra ef Burns, 1970 ; Bourbouze et Guessous, 1977). De ce fait, il est apparu nécessaire d'avoir des précisions sur les caractéristiques de la prise alimentaire des caprins, en particulier sur la définition du repas, la description des composantes comportementales pendant celui-ci, la description quantitative de la prise alimentaire et du comportement de choix vis-à-vis des aliments distribués. C'est l'objet de la présente note qui rapporte un travail réalisé sur des chèvres non attachées placées dans des cages spacieuses. Une précédente étude avait en effet montré qu'une chèvre attachée disposant d'une surface limitée dans une cage à bilan pouvait modifier considérablement son comportement alimentaire, son niveau d'ingestion et ses activités au cours de la journée.

Une étude typologique sur le comportement alimentaire du troupeau de chèvres. dont nous disposions a fait apparaître que quatre groupes se différenciaient sur les critères du niveau de consommation, de la proportion de refus ef de la vitesse d'ingestion. Quatre chèvres taries de race Alpine âgées de 2 à 4 ans, pesant de 48 à $64 \mathrm{~kg}$ et représentant l'un de ces quatre groupes, ont été sélectionnées pour participer à la présente expérience. Elles sont placées en cage, disposant de 2,60 $\mathrm{m}^{2}$ et totalement libres de leurs mouvements. Elles reçoivent du foin de luzerne distribué à volonté, deux fois par jour, à 7 et $14 \mathrm{~h}$. Elles disposent d'eau placée dans un bac, de paille de la litière renouvelée chaque jour et d'une pierre à sel. La durée d'éclairement des animaux est de $12 \mathrm{~h} /$ jour. 
Les enregistrements sont effectués à partir d'une salle d'observation totalement isolée et insonore. Les observations ont lieu trois fois par semaine pendant deux périodes de deux semaines au cours des $12 \mathrm{~h}$ d'éclairement. Le foin ingéré est mesuré grâce à une auge spéciale fixée à une balance. Ainsi, la quantité de foin ingérée peut être enregistrée après chaque repas.

\section{Résultats et discussion.}

Au cours d'un repas, la prise alimentaire de la chèvre peut s'interrompre pendant des laps de temps variables. Si ces durées excèdent $10 \mathrm{~min}$, la chèvre ne recommence à consommer que deux heures et demie plus tard, au plus tôt. Cette observation a permis de définir le repas chez la chèvre : c'est une période de temps pendant laquelle la chèvre mange, sans s'interrompre plus de $10 \mathrm{~min}$. Un repas dure en moyenne $53 \mathrm{~min}$, au minimum $15 \mathrm{~min}$ et au maximum $115 \mathrm{~min}$.

Pendant le repas, la chèvre interrompt l'ingestion de foin de luzerne pour aller, avec une fréquence égale, soit boire, soif consommer de la paille de la litière, soit lécher la pierre à sel. Lorsque la chèvre est dans une de ces trois activités comportementales, elle revient directement consommer du foin dans 70 cas sur 100 . Ainsi, le repas se compose surtout d'allers retours entre l'activité comportementale principale de consommation de foin et des activités «périphériques » d'ingestion de paille, de buvée ef de léchage de pierre à sel.

Le tableau 1 rapporte la quantité de matière sèche ingérée par jour. Les variations de cetfe quantité dues aux facteurs individuels sont importantes et significatives. La vitesse d'ingestion des chèrres semble plus lente que celle des moutons (Goeffroy,

\section{TABLEAU 1}

Quantité de matière sèche ingérée et caractéristiques de la prise alimentaire

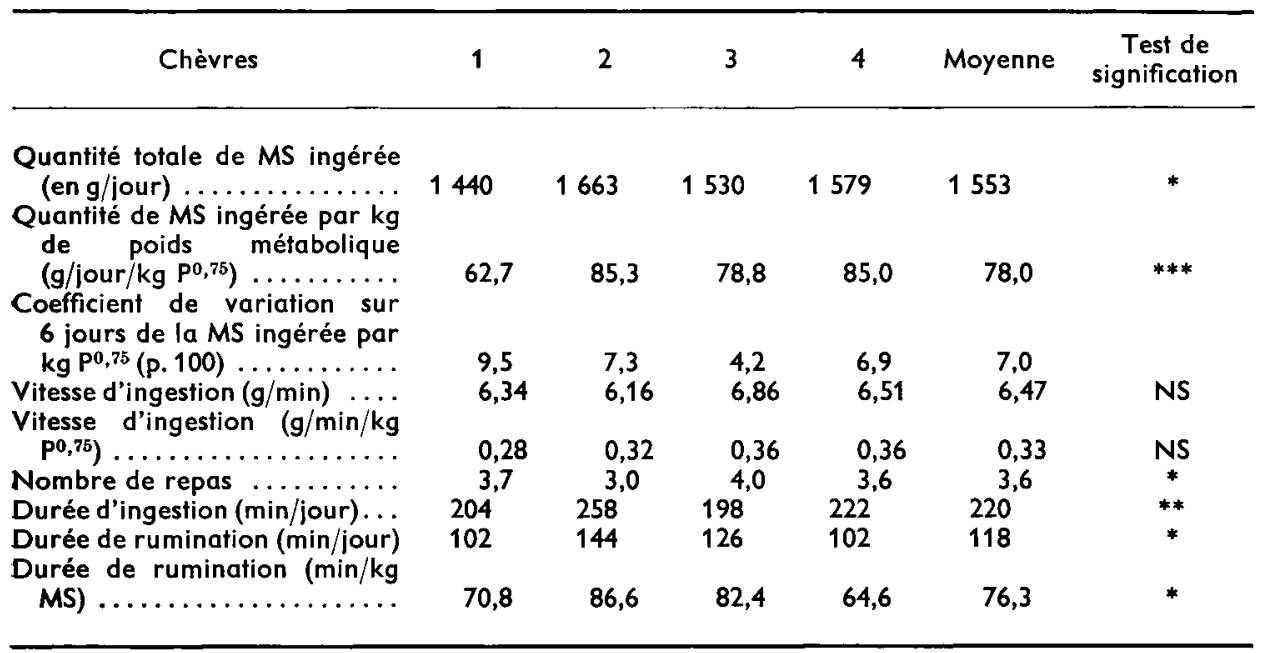
Significatif. 
1974) ; elle est peu influencée par les facteurs individuels. En revanche, les facteurs individuels ont un effet significatif sur le nombre de repas et les durées d'ingestion et de rumination. La chèvre fait des repas principaux correspondant aux distributions de foin et éventuellement des repas secondaires entre les repas principaux et pendant la nuit. En moyenne, 72 p. 100 de la matière sèche totale ingérée ont été consommés pendant les deux repas principaux et 28 p. 100 au cours des repas secondaires ef pendant la nuit. L'ingestion d'aliments dure de 3 h 20 à 4 h 20 environ, et la rumination d' 1 h 20 à 2 h 25 selon les chèvres, ce qui confirme que les caprins ruminent relativement peu le jour (Bell et Lawn, 1957 ; Goeffroy, 1974).

Par ailleurs, lorsque le foin de luzerne est distribué dans l'auge, une partie de ce foin est généralement refusé. Le foin refusé contient significativement plus de tiges et moins de feuilles qui représentent la partie du foin la plus nutritive (fig. 1). De ce

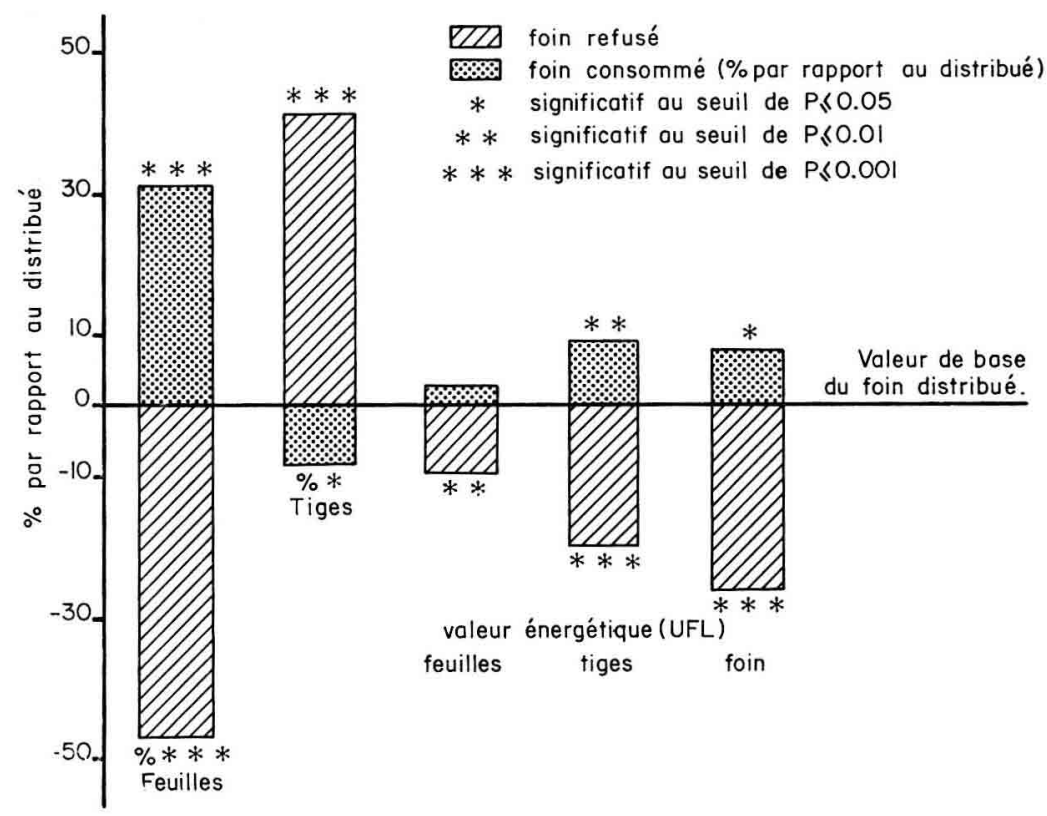

FIG. 1. - Valeur énergétique du foin de luzerne distribué, refusé et consommé par la chèvre.

fait, le foin réellement ingéré renferme plus de feuilles et moins de tiges. En outre, les feuilles refusées sont plus riches en matières minérales que les feuilles distribuées ef les tiges refusées plus riches en cellulose brute et en matières azotées que les tiges distribuées. La valeur nutritive du foin refusé exprimée en Unités Fourragères Lait sur la figure 1 (INRA, 1978) est donc significativement inférieure à celle du foin distribué. En conséquence, la valeur nutritive du foin réellement ingéré est supérieure à celle du foin distribué.

\section{Conclusion.}

Le repas d'une chèvre semble se composer d'un ensemble d'activités compre- 
nant une activité principale, l'ingestion de foin et des activités secondaires lui permettant d'équilibrer ses apports en minéraux, en eau et en parois cellulaires. La chèvre ingère lentement la ration distribuée en raison du choix très sélectif qu'elle effectue sur l'ingéré. C'est ce qui explique pourquoi la valeur nutritive du foin réellement ingéré est sensiblement supérieure à celle du foin distribué.

Journées Ingestion-Digestion-Absorption de l'Association française de Nutrition, Paris, 15-16 novembre 1979.

\section{Références}

BELL F. R., LAWN A. M., 1957. The pattern of rumination behaviour in housed goats. Brit. J. Anim. Behav., 5, 3-7.

BOURBOUZE A., GUESSOUS F., 1977. La chèvre et l'utilisation des ressources dans les milieux difficiles. Symp. La chèvre des pays méditerranéens, Malaga, Grenada, Murcia (Espagne), 3-7 octobre 1977, 207-227.

DAVENDRA C., BURNS M., 1970. Goat production in the Tropics. Commonwealth agric. Bur., Franham Royal, Bucks, England.

GOEFFROY F., 1974. Etude comparée du comportement alimentaire et mérycique de deux petits ruminants : la chèvre et le mouton. Ann. Zootech., 23, 63-73.

I.N.R.A., 1978. Alimentation des ruminants. INRA Publications, route de Saint-Cyr, 78000 Versailles, 47-87.

MORAND-FEHR P., HERVIEU J., BREMART-LE GOUSSE C., 1977. Comportement de la chèvre : réaction de l'animal vis-à-vis du fourrage distribué. Symp. La chèvre des pays méditerranéens, Malaga, Grenada, Murcia (Espagne), 3-7 octobre 1977, 156-160. 